\title{
Open
}

\section{Accurate mitochondrial DNA sequencing using off-target reads provides a single test to identify pathogenic point mutations}

\author{
Helen R. Griffin, PhD ${ }^{1}$, Angela Pyle, PhD¹, Emma L. Blakely, PhD², Charlotte L. Alston, PhD², \\ Jennifer Duff, $\mathrm{PhD}^{1}$, Gavin Hudson, $\mathrm{PhD}^{1}$, Rita Horvath, MD, PhD', Ian J. Wilson, $\mathrm{PhD}^{1}$, \\ Mauro Santibanez-Koref, PhD ${ }^{1}$, Robert W. Taylor, PhD² and Patrick F. Chinnery, PhD, FMedSci ${ }^{1}$
}

\begin{abstract}
Purpose: Mitochondrial disorders are a common cause of inherited metabolic disease and can be due to mutations affecting mitochondrial DNA or nuclear DNA. The current diagnostic approach involves the targeted resequencing of mitochondrial DNA and candidate nuclear genes, usually proceeds step by step, and is time consuming and costly. Recent evidence suggests that variations in mitochondrial DNA sequence can be obtained from whole-exome sequence data, raising the possibility of a comprehensive single diagnostic test to detect pathogenic point mutations.
\end{abstract}

Methods: We compared the mitochondrial DNA sequence derived from off-target exome reads with conventional mitochondrial DNA Sanger sequencing in 46 subjects.

Results: Mitochondrial DNA sequences can be reliably obtained using three different whole-exome sequence capture kits. Coverage correlates with the relative amount of mitochondrial DNA in the original genomic DNA sample, heteroplasmy levels can be determined using variant and total read depths, and-providing there is a minimum read depth of 20 -fold-rare sequencing errors occur at a rate similar to that observed with conventional Sanger sequencing.

Conclusion: This offers the prospect of using whole-exome sequence in a diagnostic setting to screen not only all protein coding nuclear genes but also all mitochondrial DNA genes for pathogenic mutations. Off-target mitochondrial DNA reads can also be used to assess quality control and maternal ancestry, inform on ethnic origin, and allow genetic disease association studies not previously anticipated with existing whole-exome data sets.

Genet Med advance online publication 5 June 2014

Key Words: exome; mitochondrial disorders; mitochondrial DNA; point mutation; sequencing

\section{INTRODUCTION}

Mitochondrial disorders have emerged as a common cause of inherited metabolic diseases. The underlying biochemical defect affects the respiratory chain, which is encoded by genes within mitochondrial DNA (mtDNA) and the nuclear genome. Additional nuclear-encoded mitochondrial proteins are involved in mitochondrial biogenesis, the assembly of the respiratory chain, and the maintenance of mtDNA. As a result, mutations in both nuclear DNA and mtDNA can cause mitochondrial diseases.

Mitochondrial disorders characteristically cause an overlapping spectrum of disease. With a few notable exceptions, it is very difficult to predict the genetic defect responsible in a particular individual. Having excluded a handful of common mutations, the molecular diagnostic approach is guided by the muscle histochemistry or biochemistry in an affected tissue. ${ }^{1}$ This usually involves a series of investigations aimed at excluding large-scale deletions and depletion of mtDNA, followed by targeted resequencing of the mitochondrial and nuclear genomes. ${ }^{2}$ Even with recent diagnostic advances, this can be time consuming and expensive, and only yields a molecular diagnosis in approximately two out of three patients. ${ }^{3}$ The expanding clinical spectrum means that mitochondrial disorders enter the differential diagnosis of an increasing number of patients. There is therefore a clear need to develop a more streamlined and high-yield molecular approach.

Given its small size at $\sim 16.5 \mathrm{~kb}$, the majority of laboratories use a conventional Sanger sequencing of between 20 and 40 overlapping polymerase chain reaction (PCR) fragments from each patient. Although potentially less laborious, the targeted microarrays ${ }^{4}$ remain expensive, often require extensive crossvalidation with Sanger sequencing, and have limited capacity to detect novel insertion-deletion mutations. Bespoke nextgeneration sequencing panels provide an alternative, ${ }^{5-11}$ but given the growing number of nuclear genes implicated in mitochondrial diseases, comprehensive investigation ultimately leads to whole-exome or -genome sequencing. It is therefore of great interest that mtDNA sequences have been resolved through off-capture sequencing using conventional wholeexome sequence (WES) methods. ${ }^{12-14}$ Although there have been

${ }^{1}$ Wellcome Trust Centre for Mitochondrial Research, Institute of Genetic Medicine, Newcastle University, Newcastle-upon-Tyne, UK; ${ }^{2}$ Wellcome Trust Centre for Mitochondrial Research, Institute for Ageing and Health, Newcastle University, Newcastle-upon-Tyne, UK. Correspondence: Patrick F. Chinnery (patrick.chinnery@ncl.ac.uk) 
some notable successes, ${ }^{15}$ the reliability of this approach has not been evaluated, particularly in determining mtDNA heteroplasmy. Here we compare diagnostic Sanger sequencing and three "off-the-shelf" exome capture kits. We show that, providing there is a basic minimum base coverage, whole-exome capture will reliably detect mtDNA sequence variations with a low error rate similar to that of conventional Sanger sequencing, as well as detecting mtDNA heteroplasmies.

\section{MATERIALS AND METHODS}

We studied 46 patients with suspected mitochondrial disease. All patients underwent a diagnostic workup for mtDNA disease, including long-range PCR to detect mtDNA deletions, and Sanger mtDNA sequencing. Having excluded a pathogenic mtDNA mutation, we subjected the samples to WES to investigate a suspected Mendelian mitochondrial disorder, using the same genomic DNA sample. No insertion-deletion mutations were detected by Sanger sequencing, so we restricted our analysis of off-target WES reads to single-nucleotide variants (SNVs).

\section{WES and bioinformatic pipeline}

The majority, 25 of 46, of patient DNA samples used for WES were extracted from whole blood, 8 were from muscle, and 13 were from skin fibroblasts. All DNA samples were extracted using the same standard procedure. Paired-end WES was performed using three different capture kits: SureSelect Human All Exon 50Mb Kit (Agilent, Berkshire, UK), TruSeq Exome Enrichment Kit 62Mb (Illumina, San Diego, CA), or SeqCap EZ Exome Library v2.0 (Roche Nimblegen, Madison, WI). See Supplementary Table S1 online for sample-specific details. Sequences were aligned to the human reference sequence (UCSC hg19) (http://genome.ucsc. edu/) using either BWA v0.6.2 (ref. 16) or NovoAlign v2.07.13 (http://www.novocraft.com), were formatted using Samtools v0.1.18 (ref. 17), and the duplicate reads were removed using Picard v1.75 (http://picard.sourceforge.net/). SNVs were called using Varscan v2.2.2 (ref. 18) with a minimum total coverage of 5 reads and a minimum variant read depth of 3 . The mitochondrial variants were annotated using Annovar v.21-Feb-2013 (ref. 19) and its mtDNA-specific database files (hg19_MT_GRCh37). Minor allele frequencies of variants were obtained from an inhouse group of 269 exomes and also from 15,487 full-length mitochondrial sequences in NCBI-GenBank (downloaded June 2012), which were aligned using BWA-SW ${ }^{20}$ and variants called using Varscan v2.2.2 (ref. 18). Custom Perl scripts were used to convert the hg19 mtDNA variant positions and alleles to correspond to those of the revised Cambridge reference sequence (GenBank: NC_012920.1), ${ }^{21}$ to calculate sequence read coverage depth and to combine the minor allele frequencies with the annotated variants. mtDNA haplogroups were generated using Haplogrep ${ }^{22,23}$ (http://haplogrep.uibk.ac.at/) and previously described methods. ${ }^{24}$

\section{Real-time PCR}

Real-time PCR was performed on the original DNA samples used for WES to quantify mtDNA copy number. Assessment of
mtDNA copy number was carried out using a relative, real-time PCR assay using a Bio-Rad iQ5 Optical System (Hercules, CA) as described previously. ${ }^{25}$

\section{Mitochondrial genome sequencing}

Sanger sequencing of the entire mtDNA genome was performed as described previously. ${ }^{26}$ Amplicons were sequenced using the BigDye v3.1 kit and capillary electrophoresed on an ABI3130xl Genetic Analyzer (Life Technologies, Warrington, UK). Alignment and variant calling were performed using SeqScape software (v2.1.1; Applied Biosystems, Paisley, UK), comparing with the GenBank reference sequence for human mtDNA (NC_012920.1).

\section{Assessment of mutation load by quantitative pyrosequencing}

The mtDNA mutation load for specific alleles was assessed using quantitative pyrosequencing. The Pyromark Assay Design Software v2.0 (Qiagen, Crawley, West Sussex, UK) was used to design locus-specific PCR and pyrosequencing primers (Supplementary Table S2 online) for each variant, and pyrosequencing was performed on the Pyromark Q24 platform according to the manufacturer's protocol. Quantification of the heteroplasmy level of each variant was achieved using Pyromark Q24 software to directly compare the relevant peak heights of the wild-type and the mutant nucleotides at the relevant position, as described previously. ${ }^{27}$

\section{Statistical analysis}

The correlation plot, Pearson's correlation coefficients, Poisson test statistics, and binomial exact 95\% confidence intervals (CIs) were generated using the R statistical package (v2.15.1) (http://CRAN.R-project.org/doc/FAQ/R-FAQ.html).

\section{RESULTS \\ Detecting mtDNA single-nucleotide variation in off-target WES reads}

An in-house WES analysis pipeline ${ }^{28-34}$ was used to detect SNVs in the mtDNA in genomic DNA samples from 46 patients. Exome sequences were generated using three different capture target kits (SureSelect Human All Exon 50Mb Kit (Agilent), TruSeq Exome Enrichment Kit 62Mb (Illumina), or SeqCap EZ Exome Library v2.0 (Nimblegen)) according to the manufacturers' protocols. Sequence reads were aligned to the human genome reference sequence (UCSC hg19) using either NovoAlign (http://www.novocraft.com) or BWA v0.6.2 (ref. 16) (see Supplementary Table S1 online for details). The mean permtDNA-base read depth from the WES was 130.5-fold (minimum 2.5 and maximum 4,100.3), with just under two-thirds of the 46 samples exceeding a mean per-base coverage depth of 10 -fold, and having greater than $90 \%$ of the mitochondrial genome covered to the minimum depth of 5-fold we required for variant calling (Supplementary Figure S1 online). This large overall mean depth was due to three patients (P12, P13, and P32) with exceptionally high coverage (Supplementary Table S1 
online), the exclusion of which resulted in a more typical mean read depth of 25.7-fold (minimum 2.5 and maximum 206.6) for the remaining 43 patients. As shown before, the mtDNA sequences generated by the different capture target kits showed distinct patterns of per-base coverage depth, ${ }^{14}$ whereas sequences from the same capture targets largely followed the same coverage pattern but at varying read depths (Figure 1). A total of 402 different SNVs were detected by WES; 301 SNVs were intragenic, of which 245 were within coding genes; 63 variants were nonsynonymous; and 1 variant was stop-loss (Supplementary Table $\mathbf{3}$ online). Eleven of the mtDNA variants were rare, with minor allele frequencies of less than 0.0001 , and for which the minor allele frequencies were obtained from 15,487 full-length mtDNA sequences in GenBank and mtDNA variants from 269 in-house exome sequences.

\section{Off-target read depth correlates with the relative amount of mtDNA}

Eight of the patients' exomes were generated from muscle DNA; seven of these exomes were generated using Illumina Truseq $62 \mathrm{Mb}$ capture targets and had greater than $99 \%$ of the mitochondrial genome covered to at least 5 -fold, with mean per-base depths ranging from 28 -fold to 4,100 -fold (mean $=734$-fold, $n=7$ ). The eighth "muscle" exome was the only one from this group generated using Agilent $50 \mathrm{Mb}$ capture targets and had a mean per-mtDNA base depth of 2.5 -fold with only $13.2 \%$ of bases covered to at least 5 -fold. The coverage from the "muscle" group was higher than for exomes derived from both blood and fibroblast DNA, for which the mean coverage of mtDNA showed a range from 3 -fold to 207 -fold (blood exome mean $=$ 22 -fold, $n=25$; fibroblast exome mean $=24$-fold, $n=13$ ). These findings were in keeping with previous observations, ${ }^{14}$ suggesting that off-target read depth is related to the relative number of mtDNA molecules in a given tissue sample.

Real-time PCR was used to determine the mtDNA copy number for the 46 patients (data shown in Supplementary Table S1 online). Figure 2 shows the correlation between mtDNA copy number from quantitative PCR and mean mtDNA base read depth from WES; correlations for subgroups of tissue type, capture target, and WES data batch are also shown. The Pearson's correlation coefficient $\left(R^{2}\right)$ for 40 of the patients was $0.142(n=40, P=0.382)$, in which 6 six patients were excluded from the analysis because their large values for mean coverage ( $>100$-fold) and quantitative PCR $(>500)$ were obscuring the results for the remaining samples. Restricting the analysis to the tissue subgroups increased the correlations, although they were not statistically significant (muscle: $R^{2}=0.698, n=$ 8, $P=0.054$; blood: $R^{2}=0.185, n=25, P=0.387$; fibroblast: $R^{2}=0.208, n=13, P=0.496$ ). Significant correlations were found by further restricting the analysis to patients sequenced using the Illumina Truseq $62 \mathrm{Mb}$ capture targets and WES aligned with either BWA or NovoAlign (muscle/62Mb/NovoAlign: $R^{2}=0.9913, n=5, P=9.8 \times 10^{-4}$; blood $/ 62 \mathrm{Mb} / \mathrm{BWA}: R^{2}=$ $0.880, n=13, P=7.5 \times 10^{-5}$; fibroblast $/ 62 \mathrm{Mb} / \mathrm{BWA}: R^{2}=0.951$, $n=5, P=0.013)$. However, nonsignificant correlations were found for the blood/fibroblast, Illumina Truseq $62 \mathrm{Mb}$,
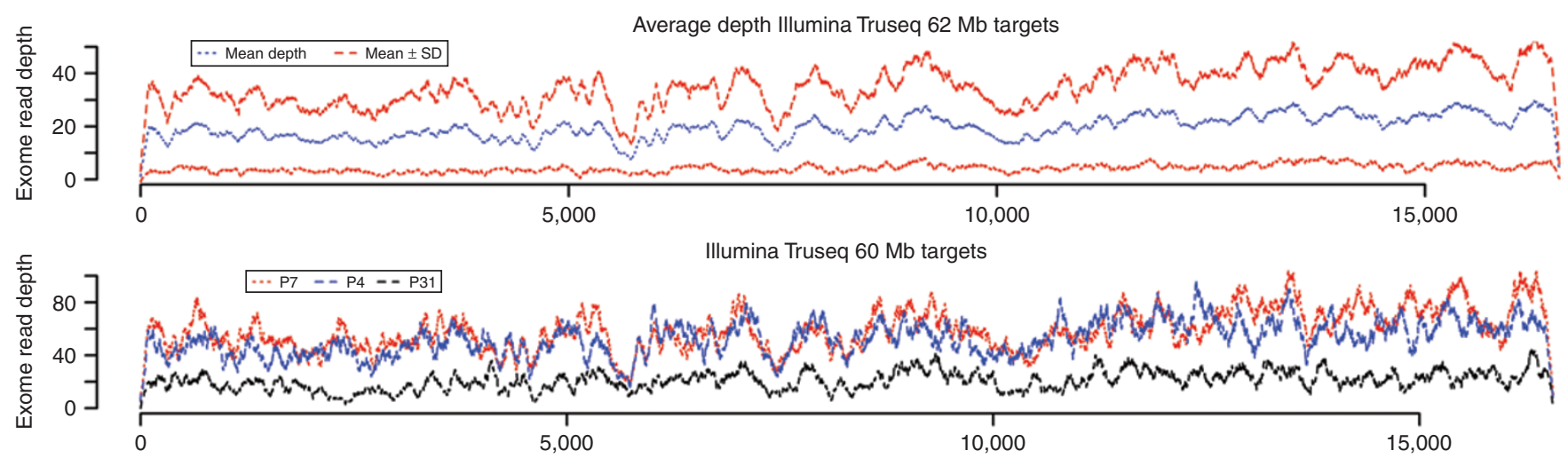

Agilent SureSelect $50 \mathrm{Mb}$ targets

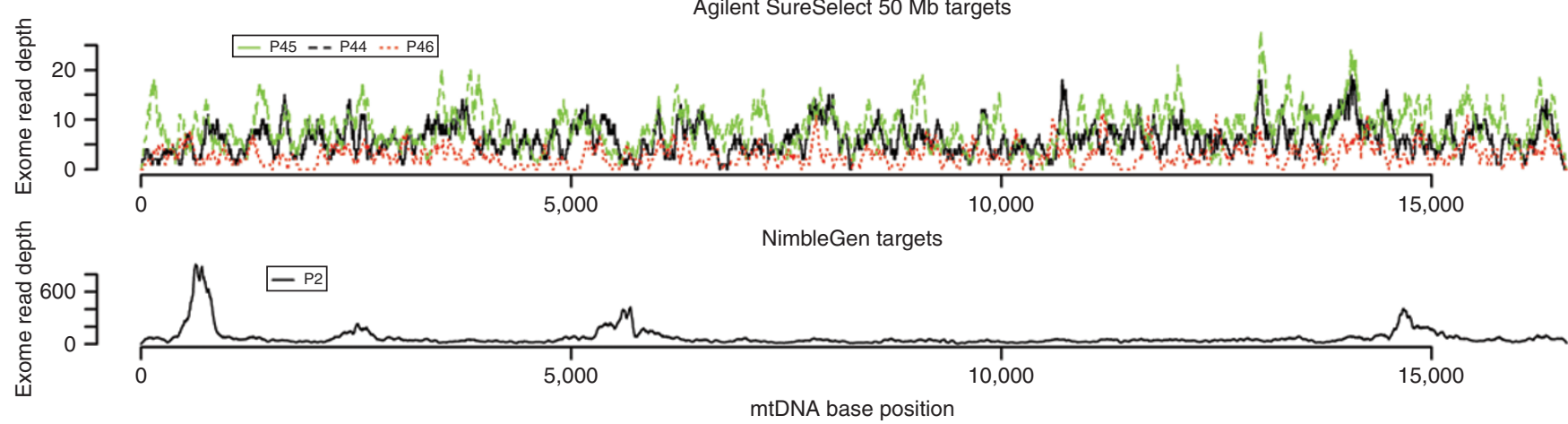

Figure 1 Average mitochondrial DNA (mtDNA) base read depth of 36 Illumina Truseq exomes and mtDNA base read depth for individual patient samples sequenced using three different whole-exome capture target kits. 

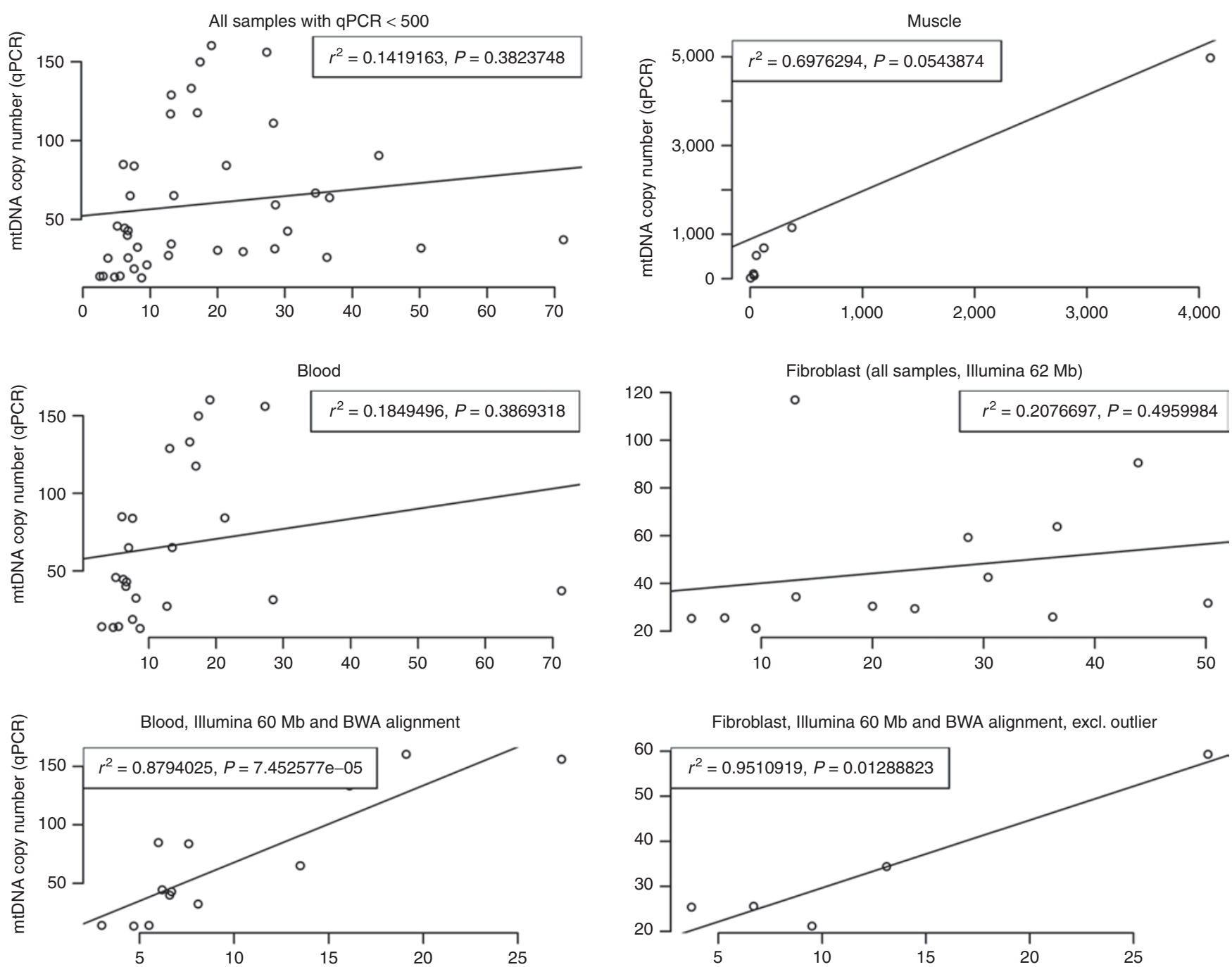

Fibroblast, Illumina $60 \mathrm{Mb}$ and BWA alignment, excl. outlier
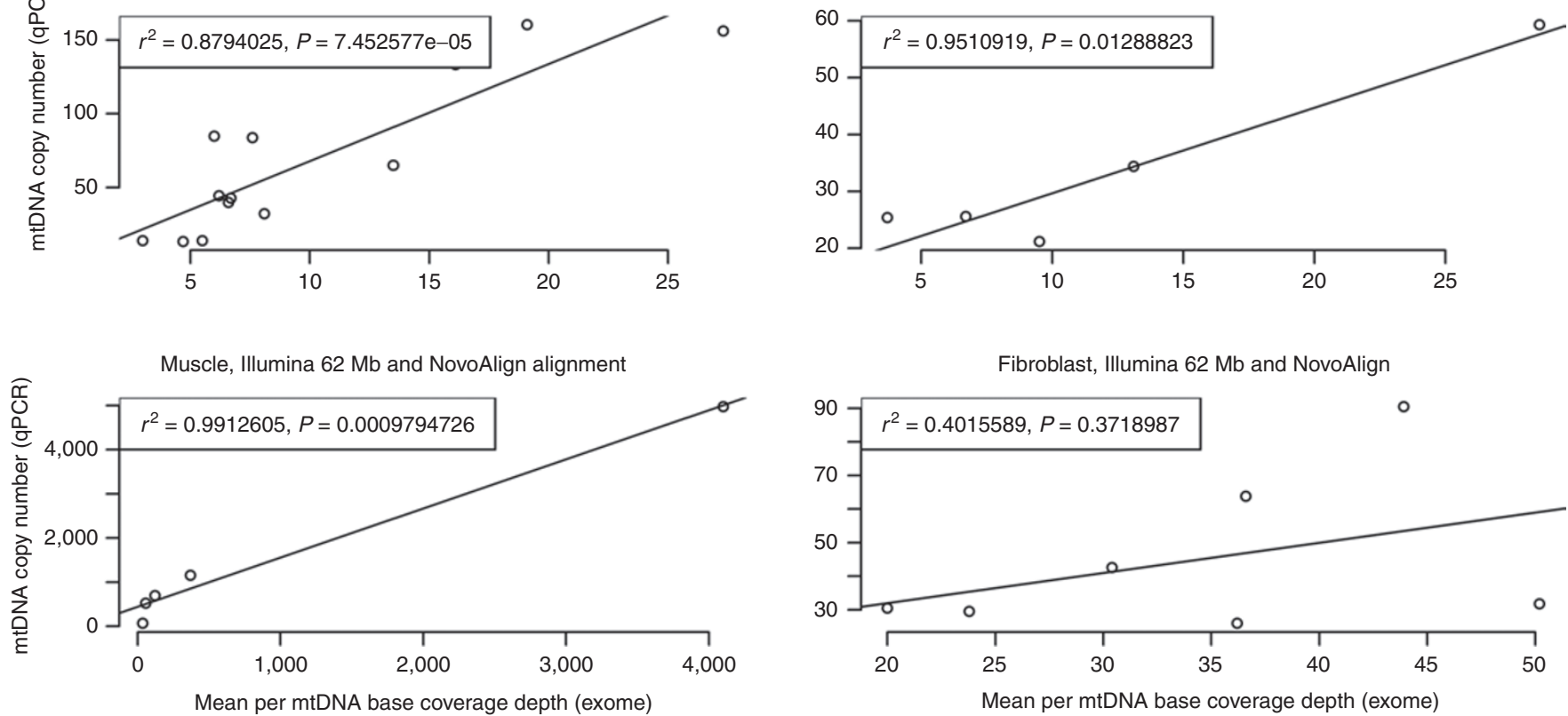

Fibroblast, Illumina $62 \mathrm{Mb}$ and NovoAlign

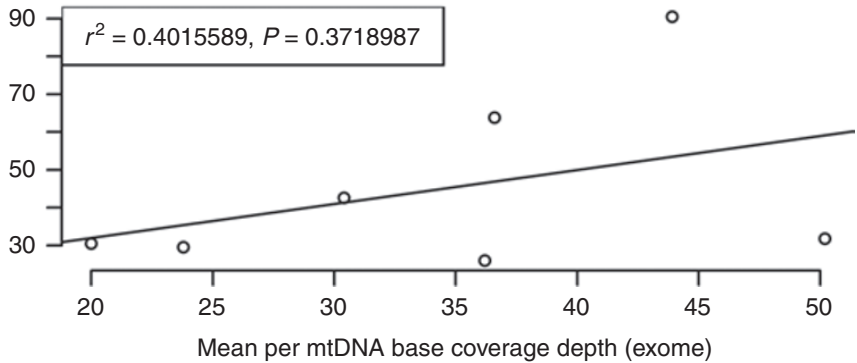

Figure 2 Correlation between mean mitochondrial DNA (mtDNA) per base depth from whole-exome sequence and mtDNA copy number from quantitative polymerase chain reaction (qPCR). qPCR measurements were performed in triplicate.

and NovoAlign subgroups (blood/62Mb/NovoAlign: $R^{2}=0.160$, $n=6, P=0.762$; fibroblast $62 \mathrm{Mb} /$ NovoAlign: $R^{2}=0.402, n=7$, $P=0.372)$. Similar correlations were obtained when the quantitative PCR data were compared directly with WES coverage in the region of the quantitative PCR amplicons. These observations confirm that, in general, off-target read depth appears to correlate with the relative amount of mtDNA, providing the
DNA was extracted from the same tissue type and WES was generated by the same protocol and analysis pipeline.

\section{Validation of WES off-target mtDNA variants by the codetection of expected haplogroup markers}

The haplogroups for each patient were predicted using all the SNV calls generated from WES for the 46 patients by using 


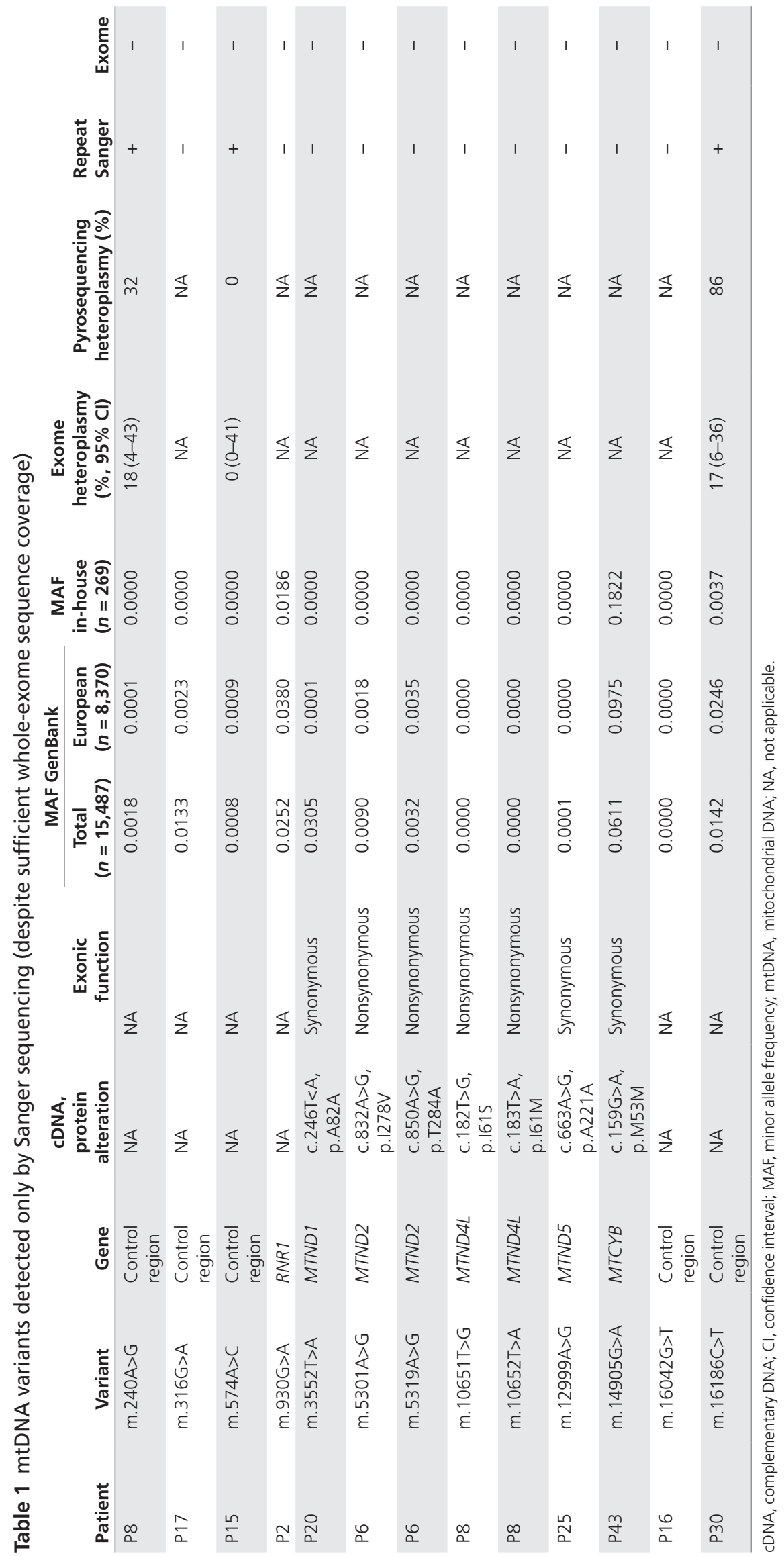




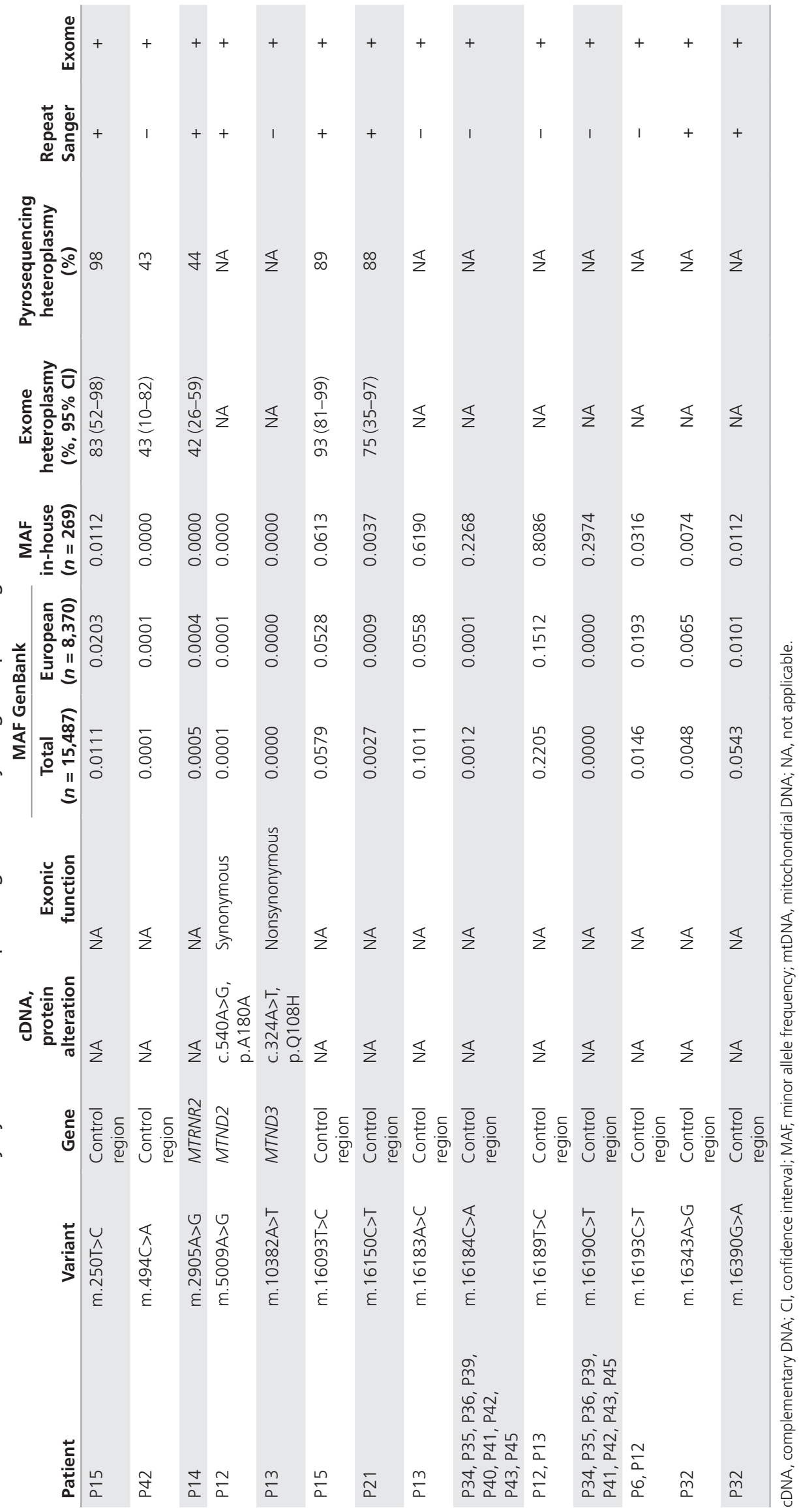



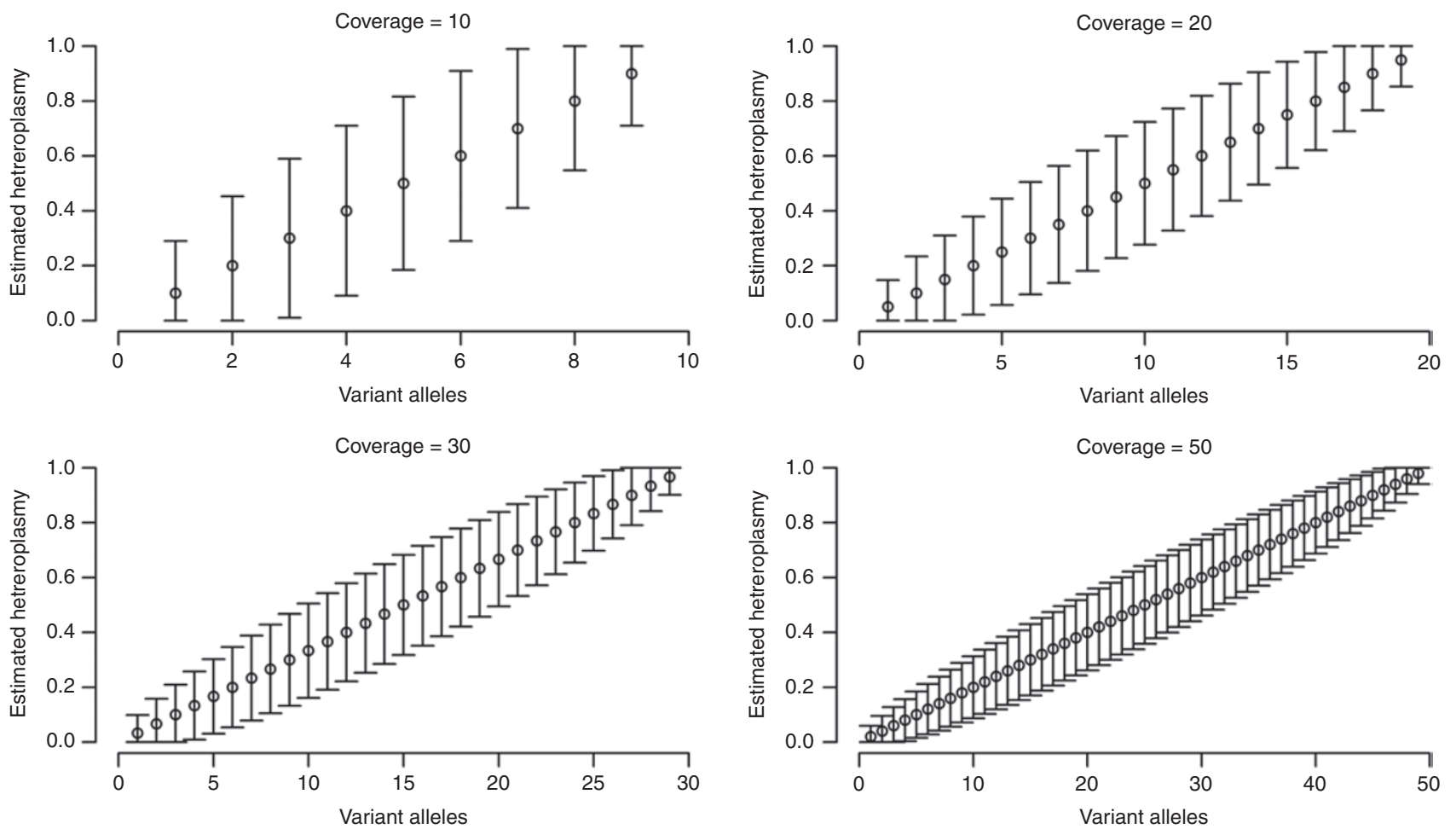

Figure 3 Binomial 95\% confidence intervals for the detection of heteroplasmies at differing whole-exome sequence coverage depths. Intervals are shown for total read coverage depths $(n)$ of 10-, 20-, 30-, and 50-fold, with the total range of variant allele counts $(v)$ from 1 to $n-1$, and the resulting estimated heteroplasmies $(\mathrm{v} / \mathrm{n})$ of between 0.0 and $1.0(0-100 \%)$. The plots demonstrate a reduction in the size of the confidence intervals at increasing exome read depths, indicating that the higher the total depth, the closer the estimated heteroplasmy level is likely to be to the true heteroplasmy level.

the Haplogrep software. ${ }^{22,23}$ The haplogroup predictions were all assigned quality scores of greater than $85 \%$, with the exception of patient P1, who was predicted to belong to haplogroup R6a but with a lower-quality score of $73 \%$. Patient haplogroup distribution (Supplementary Table S4 online) was not significantly different from that of UK controls,$^{24}$ suggesting that the SNV calls from WES appear to be genuine sequence variants and can be used to determine a patient's mitochondrial haplogroup. This may be of value in forensic and population genetic studies or as part of the quality-control procedure when analyzing WES data.

\section{Coverage threshold for the detection of WES off-target mtDNA variants}

SNVs called by the analysis pipeline were compared with variants identified using diagnostic Sanger sequencing protocols. Supplementary Table S5 online shows the number of SNVs detected in each patient for each sequencing technique relative to the revised Cambridge reference sequence (GenBank: NC_012920.1). A total of 762,174 bases of mtDNA were sequenced across the 46 patients. Sanger sequence detected SNVs at 1,277 bases, and WES detected SNVs at 1,110 bases; 1,079 SNVs were codetected by the two sequencing technologies. The vast majority of SNVs (185/198) that were detected only by Sanger sequencing corresponded to bases for which the WES read depth was less than the fivefold threshold for SNV calling. In total, 106,700 of 762,174 (14\%) bases failed to meet the fivefold calling threshold, resulting in SNVs being missed by WES in 19 patients; only 6 patients had all 16,569 base pairs of their mtDNA completely "covered" (fivefold) by WES. This led us to propose a minimum mean per-mtDNA-base coverage threshold of 20-fold in WES to ensure that the vast majority of mtDNA SNVs are detected. In our data, 20 of 46 patients reached this threshold, with 330,684 of 331,380 (99.8\%) bases sequenced to the minimum fivefold by WES and 0 of $512 \mathrm{SNVs}$ missed due to low coverage.

Tables 1 and 2 show 13 mtDNA SNVs were detected only by the original Sanger sequence, despite adequate WES read depth at each base (greater than fivefold), and 14 mtDNA SNVs were detected only by WES. Replicate Sanger sequencing was then performed from the original patient DNA across each one of these 27 variant sites. Furthermore, 10 of 13 SNVs originally detected by Sanger sequencing were not detected in the repeat Sanger sequence and were therefore likely to be sequencing artifacts from the initial Sanger sequencing. Of the remaining three SNVs, two (m.240A $>$ G and m.16186C $>$ T) could be seen in WES by manually evaluating the reads that showed potential heteroplasmy, but they had low variant base quality scores.

Pyrosequencing from the original DNA sample identified these two variants as being 32 and $86 \%$ heteroplasmic as compared to heteroplasmy predictions from WES reads of $18 \%$ (95\% CI: 4-43\%, CIs based on the binomial distribution and allele read frequencies) and 17\% (95\% CI: 6-36\%), respectively. For $\mathrm{m} .240 \mathrm{~A}>\mathrm{G}$, the $95 \% \mathrm{CI}$ for the WES heteroplasmy estimate 
(4-43\%) included the pyrosequencing result (32\%). The total WES read depth at m.240A $>$ G was 17 -fold, with 3 reads containing the variant allele ( 3 of $17=18 \%$ ). Greater read coverage depth would decrease the width of these CIs and increase the accuracy of the WES heteroplasmy estimate, as demonstrated in Figure 3. On the other hand, the 95\% CIs for the WES heteroplasmy estimate for $\mathrm{m} .16186 \mathrm{C}>\mathrm{T}(6-36 \%)$ did not contain the value obtained by pyrosequencing (86\%). m.16186C $>\mathrm{T}$ is located within a poly-C tract, which could have affected WES read alignment, thus explaining why the pyrosequencing heteroplasmy measurement was different from the WES estimate. The third Sanger-only variant $(\mathrm{m} .574 \mathrm{~A}>\mathrm{C})$ was not identified by pyrosequencing; this variant is also located next to a poly-C tract, which may be interfering with the variant detection from both WES and pyrosequencing.

Overall, the false-positive rate for Sanger sequencing was 10 of 762,174 total or $0.0013 \%$, which is in keeping with previous estimates and corresponds to the DNA polymerase fidelity. ${ }^{35}$ On the other hand, 7 of 14 SNVs (24 of 31 bases), which were only identified by WES off-target reads at $>4$-fold depth, were not seen in the repeat Sanger sequence. This corresponds to an error rate of 24 of 762,174 or $0.0031 \%$ for WES, which shows a marginally significant increase as compared to the Sanger rate (Poisson exact ratio test $(P=0.02431)$ of rate ratio $=1$ ). However, considering that the false-positive calls from WES repeatedly occurred at the same bases located in the region of the poly-C sequence tract m.16184-16193, with only two WES false calls outside of this region, the false call rate from WES for the vast majority of the mitochondrial genome is actually significantly lower than that of Sanger sequencing ( 2 of 761,714 or $0.0003 \%$ (WES) vs. 10 of 761,714 or $0.0013 \%$ (Sanger); Poisson exact ratio test $(P=0.03857)$ of rate ratio $=1)$. Of the seven SNVs that were originally identified only by WES but subsequently confirmed by repeat Sanger sequence, five were located in the noncoding control region $(\mathrm{m} .250 \mathrm{~T}>\mathrm{C}, \mathrm{m} .16093 \mathrm{~T}>\mathrm{C}$, m.16150C $>$ T, m.16343A $>\mathrm{G}$, and $\mathrm{m} .16390 \mathrm{G}>\mathrm{A}$ ) and one was a synonymous SNV in MTND2 (m.5009A $>\mathrm{G})$, and so these were assumed to be of no functional consequence. The seventh SNV, which was located in MTRNR2 (m.2905A>G) and appeared from the WES, reads to be $42 \%$ heteroplasmic (95\% CI: $26-$ 69\%). Pyrosequencing estimated the MTRNR2 variant to be $44 \%$ heteroplasmic, which fell within the confidence interval from the WES data.

\section{The detection of mtDNA heteroplasmy by off-target WES}

Next we looked for evidence of heteroplasmic SNVs in 13 of the 46 patients who had a mean mtDNA read depth greater than 30-fold, focusing on heteroplasmy levels between 10 and 90\%. All 13 of the patients had SNVs that appeared to be heteroplasmic, but all were at the same nine base positions and were therefore assumed to be false calls (Supplementary Table S6 online). Three of the patients had SNVs that appeared to show heteroplasmy unique to the individual person (Supplementary Table S7 online). Heteroplasmic variants were confirmed by pyrosequencing for three out of five of these variants, at levels that were within the 95\% CIs for the estimates from WES reads. The other two variants were detected but were homoplasmic on the pyrosequencing. These false heteroplasmy predictions from the WES reads were assumed to have occurred due to erroneous "background" variant base calls of threefold and fivefold, as compared to most base positions, which had only a "background" variant call level of between zerofold and twofold.

\section{DISCUSSION}

Here we show that off-target WES reads can be used to reliably determine the mtDNA sequence, providing there is adequate read depth. With $>20$-fold mean coverage, the error rate is only marginally higher than that of conventional diagnostic Sanger sequencing, and if the WES errors that repeatedly occur at the poly-C sequence tract are taken into account, the error rate is marginally lower in WES as compared to Sanger sequencing. Given the potential role of mtDNA mutations in a broad range of human disease phenotypes, we suggest that mtDNA analysis should be included in a diagnostic exome analysis pipeline.

Despite the exciting prospect of acquiring this potentially revealing data "gratis," any positive results should be interpreted with caution. Although only one sample was sequenced after Nimblegen capture, limiting our ability to make a formal comparison across all three capture platforms, the per-mtDNA-base coverage plots (Figure 1) generated from our study confirm previous findings describing four peaks with Nimblegen targets. ${ }^{14}$ These peaks correspond to nuclear-encoded mitochondrial sequences, leading to false mtDNA calls and potential heteroplasmy. On the other hand, here we show that nuclearencoded mitochondrial sequences do not pose a problem for the accurate calling of mtDNA SNVs and detection of heteroplasmy when using Illumina Truseq $(62 \mathrm{Mb})$ or Agilent SureSelect $(50 \mathrm{Mb})$ targeted sequence and a standard WES analysis pipeline, in which there is no need for an additional realignment of mitochondrial sequence reads to the mitochondrial genome as has been previously suggested. ${ }^{14}$ However, with both of these platforms, we observed dips in coverage in the region of the origin of light-strand replication (Ori-L, Figure 1). Consistency between different capture technologies suggests that the reduced coverage reflects the local sequence context, making these regions vulnerable to sequencing error when the mean coverage is low. Furthermore, common pathogenic mtDNA mutations are found in approximately 1 out of 300 of the general population, so any sequencing result must be interpreted in the correct clinical context. It is also worth noting that rare, region-specific polymorphic variants may not be listed on public databases. Having a population-matched control database has proven invaluable in our experience, allowing the early identification and exclusion of likely polymorphisms.

Finally, although this study was not specifically designed to determine the threshold for detecting heteroplasmy, it was found that mtDNA heteroplasmies could be detected in our data at levels of between approximately 40 and 90\% from WES when the sequence had a mean read coverage depth $>30$-fold. It remains to be seen whether WES data can be used to reliably 
detect lower levels of heteroplasmy, which is more likely to be possible at greater coverage depths, as shown in Figure 3. However, tissue-specific pathogenic mtDNA point mutations are a rare but well-recognized cause of mitochondrial disease, so the absence of a pathogenic mtDNA mutation in WES from blood DNA does not exclude the possibility of a pathogenic mtDNA point mutation in skeletal muscle. Given our finding that the WES of skeletal muscle DNA yields excellent mtDNA coverage, there is a strong argument in favor of WES of DNA extracted from a clinically affected tissue for comprehensive genetic analysis of mtDNA and relevant nuclear genes in a patient with suspected mitochondrial disease.

The reliable extraction of mtDNA sequence from WES data has several advantages. Geographically restricted mtDNA sequence motifs enable an evaluation of maternal ancestry and can thus be incorporated into quality-control algorithms when analyzing the nuclear DNA sequence. MtDNA genotyping may be of value for forensic or population genetic studies. Common mtDNA variants have been associated with several common diseases, and the acquired mtDNA sequence can be incorporated into the analysis of complex traits. Finally, given the broad clinical phenotype, mtDNA disorders enter the differential diagnosis of a wide range of diseases. Our analysis shows that WES data, aimed at the diagnosis of suspected nuclear genetic disorders, can also be used to diagnose pathogenic mtDNA mutations, providing the level of heteroplasmy is above the detection threshold. As a first-line test, currently WES would be a costly way of investigating a suspected mitochondrial disorder presenting with the classic phenotype of a specific mtDNA disease. However, as costs fall and the analysis becomes more automated, WES may become the first port of call for clinicians suspecting mitochondrial disease in the future.

\section{SUPPLEMENTARY MATERIAL}

Supplementary material is linked to the online version of the paper at http://www.nature.com/gim

\section{ACKNOWLEDGMENTS}

P.F.C. is a Wellcome Trust Senior Fellow in Clinical Science (101876/Z/13/Z), and a UK NIHR Senior Investigator, and also receives support from EU FP7 TIRCON and the National Institute for Health Research (NIHR) Newcastle Biomedical Research Centre based at Newcastle upon Tyne Hospitals NHS Foundation Trust and Newcastle University. The views expressed are those of the authors and not necessarily those of the NHS, the NIHR, or the Department of Health. P.F.C. and R.W.T. receive support from the Wellcome Trust Centre for Mitochondrial Research (096919Z/11/Z) and the Medical Research Council (UK) Neuromuscular Centre (G0601943). R.W.T. receives support from the MRC Centre for Neuromuscular Diseases (G0601943) and the UK NHS Highly Specialised "Rare Mitochondrial Disorders of Adults and Children" Service. C.L.A. is funded by a NIHR/CSO Healthcare Science Research Fellowship from the National Institute for Health Research.

\section{DISCLOSURE}

The authors declare no conflict of interest.

\section{REFERENCES}

1. McFarland R, Taylor RW, Turnbull DM. A neurological perspective on mitochondrial disease. Lancet Neurol 2010;9:829-840.

2. Greaves LC, Reeve AK, Taylor RW, Turnbull DM. Mitochondrial DNA and disease. J Pathol 2012;226:274-286.

3. DiMauro S, Schon EA, Carelli V, Hirano M. The clinical maze of mitochondrial neurology. Nat Rev Neurol 2013;9:429-444.

4. Hartmann A, Thieme M, Nanduri LK, et al. Validation of microarray-based resequencing of 93 worldwide mitochondrial genomes. Hum Mutat 2009;30:115-122.

5. Sosa MX, Sivakumar IK, Maragh S, et al. Next-generation sequencing of human mitochondrial reference genomes uncovers high heteroplasmy frequency. PLOS Comput Biol 2012;8:e1002737.

6. Li M, Schönberg A, Schaefer M, Schroeder R, Nasidze I, Stoneking M. Detecting heteroplasmy from high-throughput sequencing of complete human mitochondrial DNA genomes. Am J Hum Genet 2010;87:237-249.

7. Tang S, Huang T. Characterization of mitochondrial DNA heteroplasmy using a parallel sequencing system. BioTechniques 2010;48:287-296.

8. Holland MM, McQuillan MR, O'Hanlon KA. Second generation sequencing allows for mtDNA mixture deconvolution and high resolution detection of heteroplasmy. Croat Med J 2011;52:299-313.

9. Huang T. Next generation sequencing to characterize mitochondrial genomic DNA heteroplasmy. Curr Protoc Hum Genet 2011; Chapter 19:Unit19.8.

10. Payne BA, Wilson IJ, Yu-Wai-Man P, et al. Universal heteroplasmy of human mitochondrial DNA. Hum Mol Genet 2013;22:384-390.

11. Ramos A, Santos C, Mateiu L, et al. Frequency and pattern of heteroplasmy in the complete human mitochondrial genome. PLOS ONE 2013;8:e74636.

12. Tang S, Wang J, Zhang VW, et al. Transition to next generation analysis of the whole mitochondrial genome: a summary of molecular defects. Hum Mutat 2013;34:882-893.

13. Samuels DC, Han L, Li J, et al. Finding the lost treasures in exome sequencing data. Trends Genet 2013;29:593-599.

14. Picardi E, Pesole G. Mitochondrial genomes gleaned from human whole-exome sequencing. Nat Methods 2012;9:523-524.

15. Delmiro A, Rivera H, García-Silva MT, et al. Whole-exome sequencing identifies a variant of the mitochondrial MT-ND1 gene associated with epileptic encephalopathy: West syndrome evolving to Lennox-Gastaut syndrome. Hum Mutat 2013;34:1623-1627.

16. Li H, Durbin R. Fast and accurate short read alignment with Burrows-Wheeler transform. Bioinformatics 2009;25:1754-1760.

17. Li H, Handsaker B, Wysoker A, et al.; 1000 Genome Project Data Processing Subgroup. The Sequence Alignment/Map format and SAMtools. Bioinformatics 2009;25:2078-2079.

18. Koboldt DC, Zhang Q, Larson DE, et al. VarScan 2: somatic mutation and copy number alteration discovery in cancer by exome sequencing. Genome Res 2012;22:568-576.

19. Wang K, Li M, Hakonarson H. ANNOVAR: functional annotation of genetic variants from high-throughput sequencing data. Nucleic Acids Res 2010;38:e164.

20. Li H, Durbin R. Fast and accurate long-read alignment with Burrows-Wheeler transform. Bioinformatics 2010;26:589-595.

21. Andrews RM, Kubacka I, Chinnery PF, Lightowlers RN, Turnbull DM, Howell N. Reanalysis and revision of the Cambridge reference sequence for human mitochondrial DNA. Nat Genet 1999;23:147.

22. van Oven M, Kayser M. Updated comprehensive phylogenetic tree of global human mitochondrial DNA variation. Hum Mutat 2009;30:E386-E394.

23. Kloss-Brandstätter A, Pacher D, Schönherr S, et al. HaploGrep: a fast and reliable algorithm for automatic classification of mitochondrial DNA haplogroups. Hum Mutat 2011;32:25-32.

24. Hudson G, Panoutsopoulou K, Wilson I, et al.; arcOGEN Consortium. No evidence of an association between mitochondrial DNA variants and osteoarthritis in 7393 cases and 5122 controls. Ann Rheum Dis 2013;72:136139.

25. He L, Chinnery PF, Durham SE, et al. Detection and quantification of mitochondrial DNA deletions in individual cells by real-time PCR. Nucleic Acids Res 2002;30:e68. 
26. Taylor RW, Barron MJ, Borthwick GM, et al. Mitochondrial DNA mutations in human colonic crypt stem cells. J Clin Invest 2003;112:1351-1360.

27. White HE, Durston VJ, Seller A, Fratter C, Harvey JF, Cross NC. Accurate detection and quantitation of heteroplasmic mitochondrial point mutations by pyrosequencing. Genet Test 2005;9:190-199.

28. Dickinson RE, Griffin H, Bigley V, et al. Exome sequencing identifies GATA2 mutation as the cause of dendritic cell, monocyte, B and NK lymphoid deficiency. Blood 2011;118:2656-2658.

29. Horvath R, Holinski-Feder E, Neeve VC, et al. A new phenotype of brain iron accumulation with dystonia, optic atrophy, and peripheral neuropathy. Mov Disord 2012;27:789-793.

30. Marina AD, Schara U, Pyle A, et al. NDUFS8-related Complex I Deficiency Extends Phenotype from "PEO Plus" to Leigh Syndrome. JIMD Rep 2013;10:17-22.

31. Neeve VC, Pyle A, Boczonadi V, et al. Clinical and functional characterisation of the combined respiratory chain defect in two sisters due to autosomal recessive mutations in MTFMT. Mitochondrion 2013;13:743-748.

32. Pfeffer G, Elliott HR, Griffin H, et al. Titin mutation segregates with hereditary myopathy with early respiratory failure. Brain 2012;135(Pt 6) 1695-1713.

33. Pyle A, Griffin H, Yu-Wai-Man P, et al. Prominent sensorimotor neuropathy due to SACS mutations revealed by whole-exome sequencing. Arch Neurol 2012;69:1351-1354.
34. Talim B, Pyle A, Griffin H, et al. Multisystem fatal infantile disease caused by a novel homozygous EARS2 mutation. Brain 2013;136(Pt 2):e228.

35. Eckert KA, Kunkel TA. High fidelity DNA synthesis by the Thermus aquaticus DNA polymerase. Nucleic Acids Res 1990;18:3739-3744.

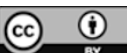

This work is licensed under a Creative Commons Attribution 3.0 Unported License. The images or other third party material in this article are included in the article's Creative Commons license, unless indicated otherwise in the credit line; if the material is not included under the Creative Commons license, users will need to obtain permission from the license holder to reproduce the material. To view a copy of this license, visit http:// creativecommons.org/licenses/by/3.0/ 